\title{
Suppression of the Intermittent Blob-type Transport by the Resonant Magnetic Perturbation (RMP) in the TEXTOR Tokamak
}

Y. Xu${ }^{1}$, R. R. Weynants ${ }^{1}$, M. Van Schoor ${ }^{1}$, M. Vergote ${ }^{1}$, S. Jachmich ${ }^{1}$, M. W. Jakubowski ${ }^{2}$, M. Mitri ${ }^{3}$, D. Reiser ${ }^{3}$, O. Schmitz ${ }^{3}$, K. H. Finken ${ }^{3}$, M. Lehnen ${ }^{3}$, B. Unterberg ${ }^{3}$, D. Reiter ${ }^{3}$, U. Samm ${ }^{3}$, and the TEXTOR team

* Partners in the Trilateral Euregio Cluster (TEC):

${ }^{1}$ Laboratory for Plasma Physics, Ecole Royale Militaire/Koninklijke Militaire School, Euratom-Belgian State Association, Avenue de la Renaissance 30, B-1000 Brussels, Belgium*

${ }^{2}$ Max-Planck-Institut für Plasmaphysik, IPP-EURATOM Association, Teilinstitut Greifswald, Wendelsteinstr. 1, 17491Greifswald, Germany

${ }^{3}$ Institute für Energieforschung-Plasmaphysik, Forschungszentrum Jülich, Association Euratom, D-52425 Juelich, Germany*

\begin{abstract}
During the static 6/2 DED (dynamic ergodic divertor) experiments at TEXTOR, a significant impact of the edge resonant magnetic perturbation (RMP) on the blob transport in the scrape-off layer (SOL) has been observed. In ohmic discharges without the RMP, the turbulence intermittency extends deep into the SOL with a radially-outward speed of $\sim 1 \mathrm{~km} / \mathrm{s}$ and ejects large turbulent flux. The fraction of the blob-carried to total turbulent flux is enhanced with increasing plasma density and reaches about $60 \%$ of the total flux in the SOL in high density discharges. With the application of the RMP, the blob size and their radially moving velocity are both reduced, resulting in a suppression of the blob transport in the SOL. It has been found that the suppression effect of the RMP on blobs is independent of the change of edge equilibrium profiles and the phasing variation of the RMP as well. The physical mechanisms appear to be consistent with the radial interchange motions of the blob structures.
\end{abstract}

PACS: $52.55 . F a$, 52.35.Ra, 52.35.Vd, 52.25.Fi

Key words: Turbulence intermittentcy, blob transport, resonant magnetic perturbation. 


\section{Introduction}

In magnetized plasmas, it has long been observed that the radial transport in the scrape-off layer (SOL) is characterized by turbulent intermittency [1-6], appearing as $\mathbf{B}$ field-aligned filaments or perpendicular-plane blobs, which unfavorably increase recycling at walls, reduce the divertor efficiency and thus may lead to a high level erosion of the first wall in fusion devices like ITER. In recent years, experiments in several machines have shown the importance of an ergodized magnetic boundary induced by the edge resonant magnetic perturbation (RMP) in mitigating the ELMs [7] and also in optimizing the plasma-wall interaction $[8,9]$. With the RMP, significant changes in edge turbulence and turbulent transport have been observed in the static DED (dynamic ergodic divertor) operation in TEXTOR [10] and in the ELM control experiments in DIII-D [11] as well. In this paper, we report the experimental results about the suppression effect of the RMP on the intermittent blob transport in the SOL of TEXTOR.

\section{Experimental set-up}

The experiments were carried out at TEXTOR tokamak mainly under following ohmic discharge conditions of deuterium plasmas: $\mathrm{R} / a=1.73 / 0.46[\mathrm{~m} / \mathrm{m}], \mathrm{B}_{\mathrm{T}}=1.9 \mathrm{~T}$, $\mathrm{I}_{\mathrm{p}}=270 \mathrm{kA}$, and chord-averaged density in a range of $<\mathrm{n}_{\mathrm{e}}>=(1.6-3.5) \times 10^{19} \mathrm{~m}^{-3}$. The edge magnetic perturbation was created by 16 DED coils, helically winding around the inner side of the torus with a pitch parallel to field lines on the magnetic flux surface with a safety factor $q \cong 3$ [9]. In the present experiment, the base poloidal/toroidal modes were operated as $m / n=6 / 2$ with a $d c$ DED current, $\mathrm{I}_{\mathrm{DED}}=6 \mathrm{kA}$, applied in the stationary phase of the ohmic discharge. The Poincare plot shows that the perturbed volume roughly covers a radial range of $\rho \equiv \mathrm{r} / a \approx 0.72-1.0$, including an ergodic zone $(\rho \approx 0.72-0.96)$ with long and a laminar zone ( $\rho \approx 0.96-1.0)$ with short field-line connection lengths, 
respectively [12]. In the experiment, the small DED current-induced edge RMP does not affect the global confinement, nor excite external tearing modes. The measurements of the turbulent intermittency were accomplished by a fast reciprocating Langmuir probe array installed at the outer midplane [10]. Two pins of the array were DC-biased to measure the ion saturation current, $\mathrm{I}_{\mathrm{s}}$, while the other two were poloidally separated to detect the poloidal electric field $\mathrm{E}_{\theta}$ from their floating potential difference. The radial speed of the blobs is hence computed by $\mathrm{V}_{\mathrm{r}}=\mathrm{E}_{\theta} \times \mathrm{B} / \mathrm{B}^{2}$. The turbulent particle flux, $\widetilde{\Gamma}_{r}$, is

calculated by $\tilde{\Gamma}_{r}=\left\langle\tilde{n} \widetilde{E}_{\theta}\right\rangle / \mathrm{B}$, where $\tilde{n}$ and $\tilde{E}_{\theta}$ are density and poloidal electric field fluctuations and $\tilde{n}$ is taken from $\mathrm{I}_{\mathrm{s}}$ fluctuations. The fluctuation data were sampled at a rate of $500 \mathrm{kHz}$. For the study of the RMP impact on the blob transport in the SOL, we plunged the fast probes several times in one discharge for comparison: one plunge before the DED and the other in the plateau of the DED phase. For each plunge, the probe position reaches the plasma edge inside the limiter location. In this study, we simply assume that the fluctuating $\mathrm{I}_{\mathrm{s}}$ represents density fluctuations.

\section{Results and discussion}

Figure 1 illustrates the basic features of the intermittent blob transport in the ohmic discharge phase before the DED. Shown in Fig. 1(b) is the raw signal of $I_{s}$ measured by the fast probe in a radial range of $1.1>\mathrm{r} / a>0.98$, including the SOL and the edge. In the $\mathrm{I}_{\mathrm{s}}$ signal, one can see a lot of intermittent "bursts", extending 4-5 cm deep into the SOL. This intermittency implies a departure from a random diffusive process in fluctuations. To be more quantitative, the corresponding third (skewness) and fourth (kurtosis) order moments of the $I_{s}$ signal are plotted in Fig. 1(a). The skewness $(S)$ and kurtosis $(K)$ reflect the degree of "asymmetry" and "peakedness" of a distribution of $\mathrm{I}_{\mathrm{s}}$ fluctuations $[4,6]$. For a Gaussian signal, $S=K=0$, whereas for others the deviation from 0 indicates a higher 
degree of non-Gaussianity. Apparently, the increase of the $S$ and $K$ values from the edge to the SOL indicates that the intermittency behaves mainly in the SOL while in the edge the fluctuations are close to Gaussian statistics. To have a closer view on the blob-like bursty behaviors, we plot a zoom of $\mathrm{I}_{\mathrm{s}}$ for a $5 \mathrm{~ms}$ time window in Fig. 1(c), together with corresponding time traces of $\mathrm{V}_{\mathrm{r}}$ and $\widetilde{\Gamma}_{r}$ signals in (d) and (e), respectively. In Figs. 1(c)-(e), we can see that at almost each density "spike" (marked by red circles), there exists a burst of radial speed, $\mathrm{V}_{\mathrm{r}} \sim 1 \mathrm{~km} / \mathrm{s}$, which eventually induces a large ejection of radial flux $\tilde{\Gamma}_{r}$. The time-asymmetry in each $\mathrm{I}_{s}$ burst, i. e., quick jump and slow decaying, suggests a radially moving blob-like structure which has a steep front and a trailing wake. As such, the fluctuation-induced transport in the SOL is often termed as "turbulent blob" transport. In the experiment, we also made a density scan $\left[(1.6-3.5) \times 10^{19} \mathrm{~m}^{-3}\right]$ and a safety factor scan $(5.8 \geqq q(a) \geqq 3.2)$. It has been found that the degree of the SOL intermittency, as indicated by $S$ and $K$ values, is enhanced with increasing plasma density and $q(a)$.

In order to further identify the importance of the blob-type transport, the contribution of the blob-carried flux to the total turbulent flux, $\Gamma_{\text {int }} / \Gamma_{\text {tot }}$, has been calculated (here, $\Gamma_{\text {tot }}=\widetilde{\Gamma}_{r}$ ). By choosing different burst amplitudes on $\mathrm{I}_{\mathrm{s}}$ (usually normalized by the standard deviation, RMS) for different burst levels of blobs, various values of $\Gamma_{\text {int }} / \Gamma_{\text {tot }}$ can be obtained. The radial profiles of $\Gamma_{\mathrm{int}} / \Gamma_{\text {tot }}$ measured in different plasma density discharges are shown in Fig. 2. In the figures, the $\Gamma_{\text {int }}$ is computed by selecting intermittent bursts larger than a threshold value in the $I_{s}$ signal, i. e., burst amplitudes $\geqq 2.5 \times$ RMS. Several features can be seen: (i) Compared to the edge, the fraction of blob-carried flux is generally higher in the SOL, consistent with the stronger intermittency in the SOL [see Fig. 1(a)]; (ii) with the increase of the plasma density, the 
values of $\Gamma_{\text {int }} / \Gamma_{\text {tot }}$ increase; (iii) at the highest density case, the intermittent blob transport takes about $60 \%$ of the total flux in the SOL. The results imply a potential danger of blobs for the high level erosion of the first wall in the fusion reactor like ITER, where plasma parameters like density are very high.

The influence of the RMP on the SOL blob transport is depicted in Fig. 3, where the radial profiles of $\mathrm{I}_{\mathrm{s}}, \mathrm{V}_{\mathrm{r}}$ and $\tilde{\Gamma}_{r}$ detected in the SOL before (left column) and during (right column) the DED are compared. In Figs. 3(a) and (e), one can see that with RMP the amplitudes of blob bursts in $I_{s}$ are decreased. The corresponding $V_{r}$ is also slightly reduced [see (b) and (f)]. The intermittency degree in turbulent flux, $\widetilde{\Gamma}_{r}$, is largely depressed [see (c) and (g)], at the same time as the time-averaged turbulent flux reduces, as shown in (d) and (h). The results clearly indicate a suppression effect of the RMP on the turbulent blob transport in the SOL, in agreement with recent simulations [13].

To understand the mechanisms, we first compared the radial profiles of the skewness and kurtosis of the $\mathrm{I}_{\mathrm{s}}$ signal before and during the DED. It is found that both of $S$ and $K$ change little or even slightly increase during the DED, indicating that the bursty feature of $I_{s}$ remains with the RMP. To gain more insight into the blob structure affected by the RMP, we have made an auto-conditional averaging of the intermittent bursts on $\mathrm{I}_{\mathrm{s}}$ data by selecting the burst amplitudes larger than two times of the standard deviation. The technical details of the statistics have been described earlier [2, 4, 6]. The conditional average of $I_{\mathrm{s}}$ fluctuations measured around $\mathrm{r} / a=1.03$ before and during the DED are shown in the inset of Fig. 3(a) and (e), respectively. It can be seen that (i) before and during DED, the burst shape is similar, suggesting a small change of $S$ and $K$ on $\mathrm{I}_{\mathrm{s}}$ fluctuations, as calculated; (ii) the burst amplitude is strongly reduced by the DED; (iii) the radial "passing time" of the blobs at the probe pin, say width-at-half-height of the burst, is nearly halved from 60 to $30 \mu$ s at that location. Since the "passing time" and $V_{\mathrm{r}}$ 
both decrease during the DED, the blob size of the radial dimension $(\approx$ "passing time" $\times$ $\mathrm{V}_{\mathrm{r}}$ ) is reduced by the RMP. The decrease of the burst amplitude by RMP is therefore linked to the reduction of the blob size. Here, we neglect the influence of a second or third dimension of the blob structures on the radial "passing time" of the blobs assuming that the local dynamic process of the blobs is dominated by the "radial ejection" resulting from the curvature-driven interchange instability [14].

In the experiment, it has been found that with RMP the edge plasma profile can be changed differently, depending on discharge parameters and the wall-condition as well. In one case, the edge density (pressure) profile decreases when RMP turns on, as displayed for the case in Fig. 3. In another case, the edge density (pressure) profile increases. To rule out that the decrease of the blob size in the SOL is caused simply by a shift of the density gradient moving deeper in, we have presented the data of another type of discharges where the edge density profile increases during DED, as shown in Figs. 4(a) and (c). From Figs. 4(a) to (d), one can see that in the SOL, the blob amplitudes and associated transport are also suppressed, similar to those in Fig. 3. The results reveal that the suppression effect of RMP on the SOL blob size and related transport is independent of the edge profile variation.

In this investigation, we also tested the phasing effect of the RMP on the blob transport detected by the probe at a toroidally localized position. For this purpose, we have made a density scan $\left[(1.6-3.5) \times 10^{19} \mathrm{~m}^{-3}\right]$, a safety factor scan $(5.8 \geqq q(a) \geqq 3.2)$ and reversed $B_{T}\left(I_{p}\right)$. In these configurations, the local magnetic topology or the phasing of RMP in front of the probe (inside limiter) is very different, as seen in the Poincare or laminar plot [12]. However, in all cases, the blob size and associated transport detected by the probe in the SOL are reduced. The tendency is the same, independent of the phasing variation of RMP. As examples, the suppression effect of RMP on the SOL 
intermittent event size is shown in Figs. 4(e, f) and (g, h) for a higher $q(a)=5.8\left(I_{p}\right.$ reduced to $200 \mathrm{kA}$ ) and a reversed $\mathrm{B}_{\mathrm{T}}\left(\mathrm{I}_{\mathrm{p}}\right)$ experiment, respectively. The results point to a global effect of the RMP on the SOL blob dynamics.

In this study, we have compared the experimental results with a possible theoretical mechanism based on the interchange instability [14]. The theory proposes that the blob structure develops on the base of a turbulent eddy inside (near) the separatrix, and is then driven radially outwards by the curvature drift on a dipolar vorticity. This hypothesis is supported by some of our observations, for example, (i) the intermittency increases with increasing plasma density and $q(\mathrm{a})$, implying a stronger driver by density gradient and longer stay of blobs in the bad curvature zone, respectively; (ii) the reversed $\mathrm{B}_{\mathrm{T}}$ experiments clearly testify the $\mathbf{B}$ curvature-polarization dynamics, as observed also in DIII-D [5]. The impact of the RMP on turbulence structures could be multifold. In simulation [13], it is shown that before RMP the modes with $\mathrm{k}_{/ /} \approx 0$ on rational surfaces are subject to strong instabilities whereas during RMP the modes are damped by resistivity because $\mathrm{k}_{/ /}$becomes finite and nonzero. With RMP, the experimental measurements $[10,15]$ also showed a strong reduction of the large-scale turbulence structures in the ergodic zone. Consequently, the blob size, based on large turbulence structures, is reduced by the RMP when the blobs generate and pass radially through the ergodic layer. The influence of the RMP on the blob moving speed may be related to an increased sheath dissipation in the perturbed volume [14]. With RMP, the effective field line connection length at the plasma boundary inside the limiter is in general shortened, leading to an increase of the sheath dissipation and thus a reduction of the radial drift velocity, as seen in Fig. 3.

\section{Conclusion}


The influence of the RMP on the turbulent blob transport has been investigated in the SOL of TEXTOR during the 6/2 DED experiment. In the ohmic phase without the RMP, the blob bursts extend 4-5 $\mathrm{cm}$ deep into the SOL with a radially-outward moving speed of $\sim 1 \mathrm{~km} / \mathrm{s}$ and eject large turbulent flux. The fraction of the blob-carried flux to the total turbulent one is enhanced with increasing plasma density and reaches about $60 \%$ in the SOL in high density discharges. With the application of the RMP, a significant influence on the SOL blob transport has been observed: the blob amplitudes and their radially moving speed are both reduced, leading to a suppression of the blob transport in the SOL. The suppression effect by RMP is independent of the edge equilibrium profile changes and the phasing variation of the RMP as well. The physical mechanisms appear to be consistent with the paradigm of the radial interchange motions of the blob structures. The results provide new evidence for a controlling role of the RMP on the edge transport, and hereby, possible implications for the optimization of the plasma-wall interaction in ITER.

\section{References}

[1] S. J. Zweben, Phys. Fluids 28, 974 (1985).

[2] A. V. Filippas et al., Phys. Plasmas 2, 839 (1995).

[3] B. LaBombard, Nucl. Fusion 40, 2041 (2000).

[4] G. Y. Antar et al., Phys. Rev. Lett. 87, 065001 (2001).

[5] J. A. Boedo et al., Phys. Plasmas 8, 4826 (2001).

[6] Y. Xu et al., Plasma Phys. Control. Fusion 47, 1841 (2005).

[7] T. E. Evans et al., Phys. Rev. Lett. 92, 235003 (2004) ; Y. Liang et al., Phys. Rev. Lett. 98, 265004 (2007).

[8] Ph. Ghendrih et al., Nucl. Fusion 42, 1221 (2002). 
[9] K. H. Finken et al., Plasma Phys. Control. Fusion 46, B143 (2004) ; M. Lehnen et al., Plasma Phys. Control. Fusion 47, B237 (2005).

[10] Y. Xu et al., Phys. Rev. Lett. 97, 165003 (2006); Y. Xu et al., Nucl. Fusion 47, 1696 (2007).

[11] R. A. Moyer et al., Phys. Plasmas 12, 056119 (2005) ; J. A. Boedo et al., Report GA-A25852 (2007).

[12] M. W. Jakubowski et al., Phys. Rev. Lett. 96, 035004 (2006).

[13] D. Reiser, Phys. Plasmas 14, 082314 (2007).

[14] O. E. Garcia et al., Phys. Plasmas 13, 082309 (2006); D. A. Russell et al., Phys. Rev. Lett. 93, 265001 (2004).

[15] J. Payan et al., Nucl. Fusion 35, 1357 (1995). 


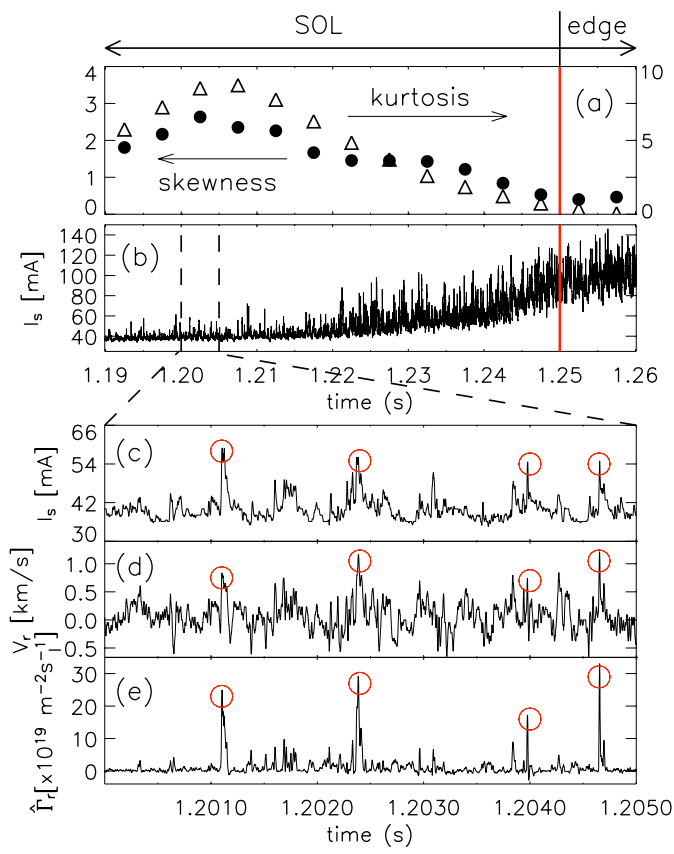

Fig. 1 Illustration of intermittent blob transport in the ohmic phase before DED (\# 101798). Time trace of $\mathrm{I}_{\mathrm{s}}$ signal measured in the scrape-off layer (SOL) and edge (b), and corresponding skewness (solid circles) and kurtosis (triangles) of the $I_{s}$ data (a). Shown in (c)-(e) are zooms of $\mathrm{I}_{\mathrm{s}}, \mathrm{V}_{\mathrm{r}}$ and $\tilde{\Gamma}_{r}$ signals in a $5 \mathrm{~ms}$ time window.

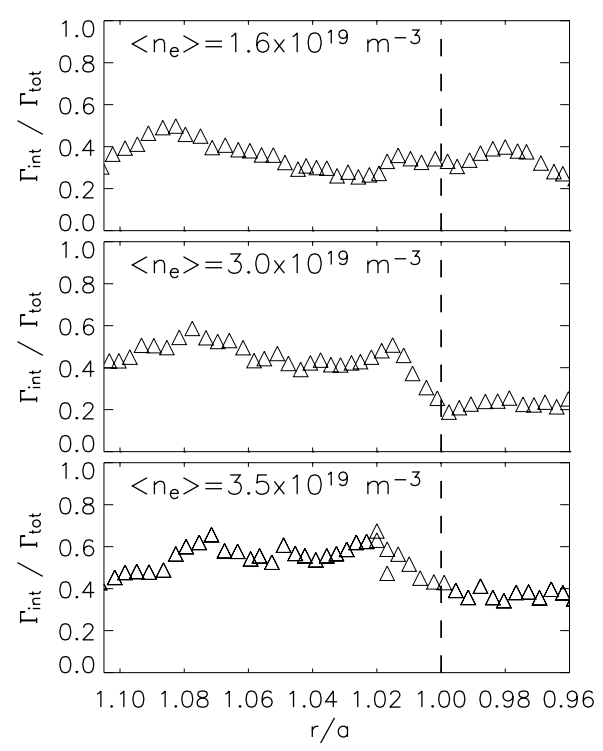

Fig. 2 Radial dependence of the ratio of blob-carried to total turbulent flux at a threshold of $\mathrm{I}_{\mathrm{s}}$ burst-amplitude/RMS $\geqq 2.5$ in three different plasma density discharges before DED. The vertical dashed lines denote limiter position. 

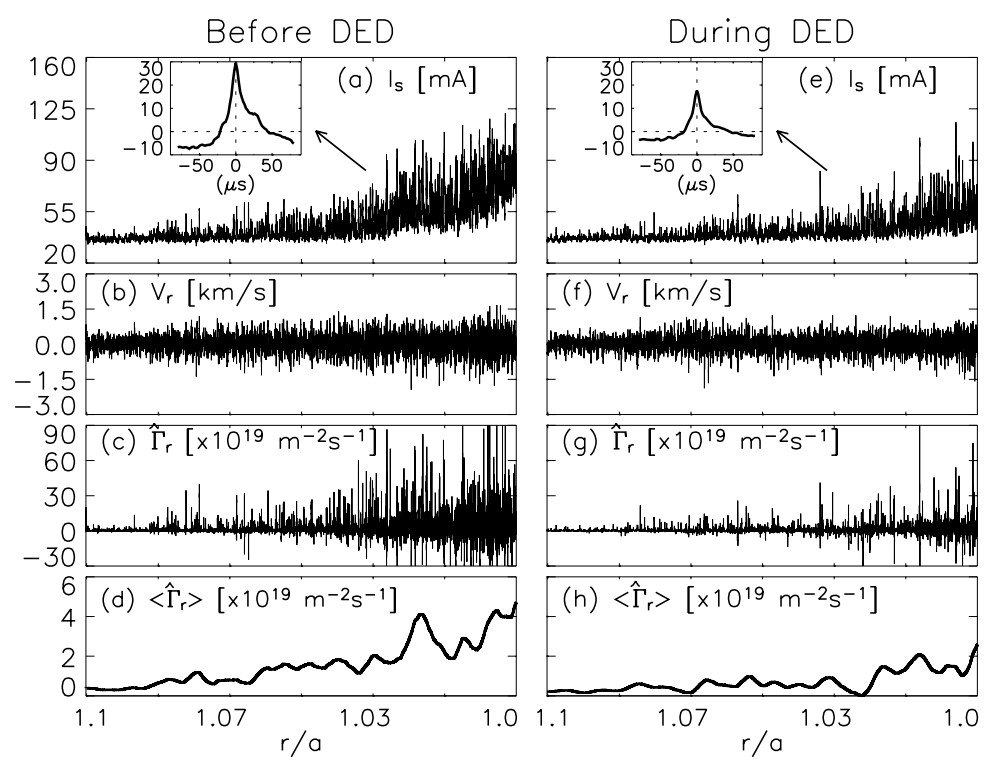

Fig. 3 Comparison of the $\mathrm{I}_{\mathrm{s}}, \mathrm{V}_{\mathrm{r}}$ and $\tilde{\Gamma}_{r}$ signals (versus normalized radial position) measured in the SOL before (left column) and during (right column) the DED (\# 101795). Plotted in (d) and (h) are the ensemble-averaged turbulent flux. Corresponding parameters in the two columns are drawn in the same scale. The inset in (a) and (e) shows the conditional-average of $\mathrm{I}_{\mathrm{s}}$ measured around $\mathrm{r} / \mathrm{a}=1.03$ before and during the DED, respectively.
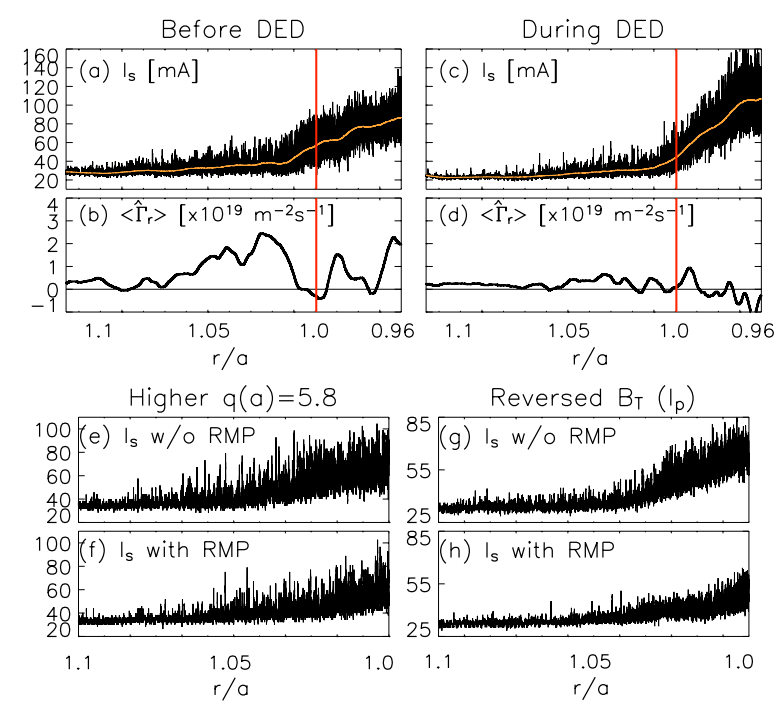

Fig. 4 (a)-(d) Comparison of $I_{s}$ and turbulent flux signals in the SOL and the edge (vertical red lines denote limiter location) before (left column) and during (right column) the DED in a discharge where the edge density $\left(I_{s}\right)$ gradient increases during the DED (\# 99630). (e, f) and (g, h) show the suppression effect of RMP on the SOL intermittent event size in a higher $q(a)=5.8$ (\# 101800) and a reversed $B_{T}\left(I_{p}\right)$ (\# 101819) experiment, respectively. 